\title{
Field-theoretical renormalization group analysis for the scaling exponents of star polymers
}

\author{
Ch. von Ferber ${ }^{1}$, Yu.Holovatch ${ }^{2,3}$ \\ 1 Theoretische Polymerphysik, Universität Freiburg, \\ D-79104 Freiburg, Germany \\ 2 Institute for Condensed Matter Physics \\ of the National Academy of Sciences of Ukraine, \\ 1 Svientsitskii Str., 79011 Lviv, Ukraine \\ 3 Ivan Franko National University of Lviv, \\ 79005 Lviv, Ukraine
}

Received September 18, 2001

\begin{abstract}
We review recent results of the field theoretical renormalization group analysis on the scaling properties of star polymers. We give a brief account of how the numerical values of the exponents governing the scaling of star polymers were obtained as well as provide some examples of the phenomena governed by these exponents. In particular we treat the interaction between star polymers in a good solvent, the Brownian motion near absorbing polymers, and diffusion-controlled reactions involving polymers.
\end{abstract}

Key words: star polymers, star exponents, renormalization group

PACS: $64.60 . A k, 61.41 .+e, 64.60 . F r, 11.10 . G h$

\section{Star exponents in polymer theory}

It is well known that the asymptotic properties of a flexible polymer chain in a good solvent are universal in the limit of infinite chain length. Lowering the temperature in a system of a polymer in a good solvent the so called $\Theta$ temperature may be reached below which the polymer collapses. At the transition temperature, the effective two point attractive and repulsive interactions between different monomers compensate each other and as a result the polymer chain may be described by a random walk (up to higher order corrections): the mean square distance between the chain endpoint $\left\langle R^{2}\right\rangle$ scales with the number of monomers $N$ as $\left\langle R^{2}\right\rangle \sim N$. Above the $\Theta$ temperature the effective interaction between the monomers is repulsive resulting in a swelling of the polymer coil which in the asymptotics again obeys a universal 
scaling law:

$$
\left\langle R^{2}\right\rangle \sim N^{2 \nu}, \quad N \rightarrow \infty
$$

with the universal exponent $\nu$ depending on the dimension of space $d$ only. The number of configurations $Z$ of a polymer chain scales with $N$ like

$$
Z \sim \mathrm{e}^{\mu N} N^{\gamma-1}, \quad N \rightarrow \infty
$$

with a non-universal fugacity $\mathrm{e}^{\mu}$ and universal scaling exponent $\gamma$.

In the early 70-ies, following the work of de Gennes [1], the analogy between the asymptotic properties of long polymer chains and the long-distance correlations of magnetic systems in the vicinity of the 2 nd order phase transition was recognized and elaborated in detail. This mapping allows to define the above exponents (1), (2) as $m \rightarrow 0$ limits of the correlation length critical exponent $\nu$ and the magnetic susceptibility critical exponent $\gamma$ of the $O(m)$-symmetric model. In this way, the powerful tools of the field theoretical renormalization group approach to the description of critical phenomena [2] can be applied in polymer theory (see e.g. [3]). In particular, this allows to calculate the exponents at $d=3$ with record accuracy [4]:

$$
\nu(d=3)=0.5882 \pm 0.0011 \quad \text { and } \quad \gamma(d=3)=1.1596 \pm 0.0020 .
$$

In dimension $d=2$, the exact values $\nu=3 / 4$ and $\gamma=43 / 32$ are known due to a mapping of two-dimensional (2D) polymers to the 2D Coulomb gas problem [5].

Under similar conditions (i.e. in good solvents above the $\Theta$ temperature and long polymer chains), star polymers [6] also obey universal scaling laws. For the homogeneous star polymer, the asymptotic properties are uniquely defined by the number of its constituting chains and by the dimension of space [7]. For the partition function (number of configurations) $Z_{f}$ of a polymer star of $f$ chains, each consisting of $N$ monomers, one finds:

$$
Z_{f} \sim \mathrm{e}^{\mu N f} N^{\gamma_{f}-1} \sim(R / \ell)^{\eta_{f}-f \eta_{2}}, \quad N \rightarrow \infty .
$$

The second part shows the scaling in terms of the size $R \sim N^{\nu}$ of the isolated coil of $N$ monomers on some microscopic length $\ell$, omitting the fugacity factor. The exponents $\gamma_{f}, \eta_{f}, f=1,2,3, \ldots$ constitute families of 'star exponents', which depend on the number of arms $f$ in a nontrivial way. The case of linear polymer chains is included in this family with the exponent $\gamma=\gamma_{1}=\gamma_{2}$ defined in (2).

Apart from $\eta_{f}$ and $\gamma_{f}$ other sets of star exponents my be defined that govern the scaling of different physical properties of polymer stars. However, all these sets of exponents can be expressed in terms of a given set by familiar scaling relations [7]. Moreover, the scaling properties of polymer networks of arbitrary but fixed topology are uniquely defined by its constituting stars [7], as long as the statistical ensemble respects some conditions on the chain length distribution [8]. Thus, the knowledge of the set of star exponents $\gamma_{f}$ or $\eta_{f}$ allows to obtain the power laws corresponding to (4) also for any polymer network of arbitrary topology [7-9]. Recently, the theory has 
also been generalized to the case of star polymers and networks that contain chains of different species, introducing additional independent families of star exponents $[9,11,12]$.

As it is usual in the theory of critical phenomena, the space dimension $d$ is crucial for the scaling laws (4). At $d=2$, there exist exact formulas expressing values of the scaling exponents of the star polymers as functions of the number of arms [10-12]. In $d=3$ dimensions on the other hand the problem does not allow for an exact solution and different approximate schemes are used. The so-called cone approximation takes into account the fact that for large $f$ each chain of the star is restricted approximately to a cone of space angle $\Omega_{f}=4 \pi / f$. In this approximation one finds for large $f[13]$

$$
\gamma_{f}-1 \sim-f^{3 / 2}
$$

However, the most accurate values of critical exponents for $d=3$ are obtained by the field theoretical renormalization group approach refined by a resummation of asymptotic series. Just in this way high precision results for the exponents governing different critical phenomena are derived [2], and this is the way the scaling exponents (3) for a polymer chain in $d=3$ were obtained. In this review, we give a brief account on how the numerical values of the star exponents were obtained for $d=3$ by means of these techniques as well as we provide some examples for phenomena governed by the star exponents.

The paper is arranged as follows. In the next section 2 we give a field-theoretical description of a star polymer in $d$ dimensions, the exponents governing scaling of a homogeneous star polymer are evaluated numerically in section 3. The theory is generalized to the case of a polymer star consisting of two species of polymers (a copolymer star) in section 4 . The following sections of the review are devoted to the numerical description of different phenomena where star exponents come to play: the interaction between polymer stars in a good solvent (section 5), the Brownian motion near absorbing polymers (section 6), diffusion-controlled reactions in presence of polymers (section 7). We finally give some conclusions and an outlook in section 8.

\section{Renormalization of polymer stars}

To give a field-theoretical description of a star polymer let us introduce an Edwards model of continuous polymer chain [3] generalized to describe a set of $f$ polymer chains of varying composition, possibly tied together at their end points. The configuration of one polymer is given by a path $r^{a}(s)$ in $d$-dimensional space $\mathbb{R}^{d}$ parametrized by a surface variable $0 \leqslant s \leqslant S_{a}$. Let us allow for a symmetric matrix of excluded volume interactions $u_{a b}$ between chains $a, b=1, \ldots, f$. The Hamiltonian $\mathcal{H}$ is then given by

$$
\frac{1}{k_{\mathrm{B}} T} \mathcal{H}\left(r^{a}\right)=\sum_{a=1}^{f} \int_{0}^{S_{a}} \mathrm{~d} s\left(\frac{\mathrm{d} r^{a}(s)}{2 \mathrm{~d} s}\right)^{2}+\frac{1}{6} \sum_{a, b=1}^{f} u_{a b} \int \mathrm{d}^{d} r \rho_{a}(r) \rho_{b}(r),
$$


with densities $\rho_{a}(r)=\int_{0}^{S_{a}} \mathrm{~d} s \delta^{d}\left(r-r^{a}(s)\right)$. In this formalism the partition function is calculated as a functional integral:

$$
\mathcal{Z}_{f}\left\{S_{a}\right\}=\int \mathcal{D}\left[r^{a}(s)\right] \exp \left\{-\frac{1}{k_{\mathrm{B}} T} \mathcal{H}\left(r^{a}\right)\right\} .
$$

Here, the symbol $\mathcal{D}\left[r_{a}(s)\right]$ includes normalization such that $Z\left\{S_{a}\right\}=1$ for all $u_{a b}=$ 0 . To make the exponential of $\delta$-functions in (7) and the functional integral welldefined in the bare theory, a cutoff $s_{0}$ is introduced such that all simultaneous integrals of any variables $s$ and $s^{\prime}$ on the same chain are cut off by $\left|s-s^{\prime}\right|>s_{0}$. Let us note that the continuous chain model (6) may be understood as a limit of discrete self-avoiding walks, when the length of each step is decreasing $\ell_{0} \rightarrow 0$ while the number of steps $N_{a}$ is increasing keeping the 'Gaussian surface' $S_{a}=N_{a} \ell_{0}^{2}$ fixed. The continuous chain model (7) can be mapped onto a corresponding field theory by a Laplace transform in the Gaussian surface variables $S_{a}$ to conjugate chemical potentials ("mass variables") $\mu_{a}[14]$ :

$$
\tilde{\mathcal{Z}}_{f}\left\{\mu_{a}\right\}=\int_{0}^{\infty} \prod_{b} \mathbf{d} S_{b} \mathrm{e}^{-\mu_{b} S_{b}} \mathcal{Z}_{f}\left\{S_{a}\right\}
$$

The Laplace-transformed partition function $\tilde{\mathcal{Z}}_{f}\left\{\mu_{a}\right\}$ can be expressed as the $m=0$ limit of the functional integral over vector fields $\phi_{a}, a=1, \ldots, f$ with $m$ components $\phi_{a}^{\alpha}, \alpha=1, \ldots, m$ :

$$
\tilde{\mathcal{Z}}_{f}\left\{\mu_{b}\right\}=\left.\int \mathcal{D}\left[\phi_{a}(r)\right] \exp \left[-\mathcal{L}\left\{\phi_{b}, \mu_{b}\right\}\right]\right|_{m=0}
$$

The Landau-Ginzburg-Wilson Lagrangian $\mathcal{L}$ of $f$ interacting fields $\phi_{b}$ each with $m$ components reads

$$
\mathcal{L}\left\{\phi_{b}, \mu_{b}\right\}=\frac{1}{2} \sum_{a=1}^{f} \int \mathrm{d}^{d} r\left(\mu_{a} \phi_{a}^{2}+\left(\nabla \phi_{a}(r)\right)^{2}\right)+\frac{1}{4 !} \sum_{a, a^{\prime}=1}^{f} u_{a, a^{\prime}} \int \mathrm{d}^{d} r \phi_{a}^{2}(r) \phi_{a^{\prime}}^{2}(r)
$$

here $\phi_{a}^{2}=\sum_{\alpha=1}^{m}\left(\phi_{a}^{\alpha}\right)^{2}$. The limit $m=0$ in (9) can be understood as a selection rule for the diagrams contributing to the perturbation theory expansions which can be easily checked diagrammatically. A formal proof of (9) using the StratonovichHubbard transformation to linearize terms in (6) is given for the multi-component case in [14]. The one particle irreducible (1PI) vertex functions $\Gamma^{(L)}\left(q_{i}\right)$ of this theory are defined by:

$$
\delta\left(\sum q_{i}\right) \Gamma_{a_{1} \ldots a_{L}}^{(L)}\left(q_{i}\right)=\int \mathrm{e}^{\mathrm{i} q_{i} r_{i}} \mathrm{~d} r_{1} \ldots \mathrm{d} r_{L}\left\langle\phi_{a_{1}}\left(r_{1}\right) \ldots \phi_{a_{L}}\left(r_{L}\right)\right\rangle_{1 \mathrm{PI}, m=0}^{\mathcal{L}}
$$

The average $\langle\cdots\rangle$ in (11) is understood with respect to the Lagrangian (10) keeping only those contributions which correspond to one-particle-irreducible graphs and which have non-vanishing tensor factors in the limit $m=0$. The partition function 
$Z_{* f}\left\{S_{a}\right\}$ of a polymer star consisting of $f$ polymers of different species $1, \ldots, f$ constrained to have a common end point is obtained from (7) by introducing an appropriate product of $\delta$-functions ensuring the "star-like" structure. It reads:

$$
Z_{* f}\left\{S_{a}\right\}=\int \mathcal{D}\left[r_{a}\right] \exp \left\{-\frac{1}{k_{\mathrm{B}} T} \mathcal{H}\left(r_{a}\right)\right\} \prod_{a=2}^{f} \delta^{d}\left(\vec{r}_{a}(0)-\vec{r}_{1}(0)\right) .
$$

The vertex part of its Laplace transformation may be defined by:

$$
\begin{aligned}
\delta(p & \left.+\sum q_{i}\right) \Gamma^{(* f)}\left(p, q_{1} \ldots q_{f}\right)= \\
& =\int \mathrm{e}^{\mathrm{i}\left(p r_{0}+q_{i} r_{i}\right)} \mathrm{d}^{d} r_{0} \mathrm{~d}^{d} r_{1} \ldots \mathrm{d}^{d} r_{f}\left\langle\phi_{1}\left(r_{0}\right) \ldots \phi_{f}\left(r_{0}\right) \phi_{1}\left(r_{1}\right) \ldots \phi_{f}\left(r_{f}\right)\right\rangle_{1 \mathrm{PI}, m=0}^{\mathcal{L}},
\end{aligned}
$$

where all $a_{1}, \ldots a_{f}$ are distinct. The vertex function $\Gamma^{(* f)}$ is thus defined by an insertion of the composite operator $\prod_{a} \phi_{a}$. Its scaling properties are governed by the scaling dimension of this operator.

In the following we will be mainly interested in two different cases: (i) a polymer star consisting of chains of one species with the single interaction $u$ between them (i.e. a homogeneous polymer star) and (ii) a polymer star constituted by two species of polymers, with interactions $u_{11}, u_{22}$ between the polymers of the same species and $u_{12}=u_{21}$ between the polymers of different species (i.e. a copolymer star). In the first case (i) one can also define $\Gamma^{(* f)}$ by the insertion of a composite operator of traceless symmetry [15]. In the case (ii) the composite operator in (13) reduces to the product of two power-of-field operators with appropriate symmetry $(\phi)^{f_{1}}\left(\phi^{\prime}\right)^{f_{2}}$ each corresponding to a product of fields of the same 'species'. Nevertheless, the results are easily generalized to the case of any number of polymer species.

As is well known, ultraviolet divergences occur when the vertex functions (11), (13) are evaluated naively [2]. We apply renormalization group ( $\mathrm{RG}$ ) theory to make use of the scaling symmetry of the system in the asymptotic limit to extract the universal content and at the same time remove divergences which occur for the evaluation of the bare functions in this limit [2]. The theory given in terms of the initial bare variables is mapped to a renormalized theory. This is achieved by a controlled rearrangement of the series for the vertex functions. Several asymptotically equivalent procedures serve to this purpose. The results reviewed in the next sections were obtained by two somewhat complementary approaches: zero mass renormalization (see [2] for instance) with successive $\varepsilon$-expansion [18] and the fixed dimension massive RG approach [19]. The first approach is performed directly for the critical point. Results for critical exponents at physically interesting dimensions $d=2$ and $d=3$ are calculated in an $\epsilon=4-d$ expansion. The second approach renormalizes off the critical limit but calculates the critical exponents directly in space dimensions $d=2, d=3$.

Let us formulate the relations for a renormalized theory in terms of the corresponding renormalization conditions. Though they are different in principle for the two procedures, we may formulate them simultaneously using the same expressions. 
Note that the polymer limit of zero component fields leads to essential simplification. Each field $\phi_{a}$, mass $m_{a}$ and coupling $u_{a a}$ renormalizes as if the other fields were absent. First we introduce renormalized couplings $g_{a b}$ by:

$$
\begin{aligned}
& u_{a a}=\mu^{\varepsilon} Z_{\phi_{a}}^{-2} Z_{a a} g_{a a}, \quad a=1,2, \\
& u_{12}=\mu^{\varepsilon} Z_{\phi_{1}}^{-1} Z_{\phi_{2}}^{-1} Z_{12} g_{12} .
\end{aligned}
$$

Here, $\mu$ is a scale parameter equal to the renormalized mass at which the massive scheme is evaluated and sets the scale of the external momenta in the massless scheme. The renormalization factors $Z_{\phi_{a}}, Z_{a b}$ are defined as power series in the renormalized couplings which fulfil the following RG conditions:

$$
\begin{aligned}
Z_{\phi_{a}}\left(g_{a a}\right) \frac{\partial}{\partial k^{2}} \Gamma_{a a}^{(2)}\left(u_{a a}\left(g_{a a}\right)\right) & =1, \\
Z_{\phi_{a}}^{2}\left(g_{a a}\right) \Gamma_{a a a a}^{(4)}\left(u_{a a}\left(g_{a a}\right)\right) & =\mu^{\varepsilon} g_{a a}, \\
Z_{\phi_{1}}\left(g_{11}\right) Z_{\phi_{2}}\left(g_{22}\right) \Gamma_{1122}^{(4)}\left(u_{a b}\left(g_{a b}\right)\right) & =\mu^{\varepsilon} g_{12} .
\end{aligned}
$$

These formulas are applied perturbatively while the corresponding loop integrals are evaluated for zero external momenta in the massive approach and for external momenta at the scale of $\mu$ in the massless approach $[2,9]$. In the massive case the $\mathrm{RG}$ condition for the vertex function $\Gamma^{(2)}$ reads

$$
\left.Z_{\phi_{a}}\left(g_{a a}\right) \Gamma_{a a}^{(2)}\left(u_{a a}\left(g_{a a}\right)\right)\right|_{k^{2}=0}=\mu^{2}, \quad a=1,2 .
$$

In the case of massless renormalization, the corresponding condition reads [2]:

$$
\left.Z_{\phi_{a}}\left(g_{a a}\right) \Gamma_{a a}^{(2)}\left(u_{a a}\left(g_{a a}\right)\right)\right|_{k^{2}=0}=0, \quad a=1,2 .
$$

In order to renormalize the star vertex functions we introduce renormalization factors $Z_{* f_{1}, f_{2}}$ by

$$
Z_{\phi_{1}}^{f_{1} / 2} Z_{\phi_{2}}^{f_{2} / 2} Z_{* f_{1}, f_{2}} \Gamma^{\left(* f_{1} f_{2}\right)}\left(u_{a b}\left(g_{a b}\right)\right)=\mu^{\delta_{f_{1}+f_{2}}}
$$

With the same formalism one can also describe a star of $f$ mutually avoiding walks [16]. In this case all interactions on the same chain $u_{a a}$ vanish and only those $u_{a b}$ with $a \neq b$ remain. Then we define the appropriate renormalization for the vertex function of mutually avoiding walks (MAW):

$$
Z_{\phi_{1}}^{f / 2} Z_{(\mathrm{MAW} f)} \Gamma_{\mathrm{MAW}}^{* f}\left(u_{12}\left(g_{a b}\right)\right)=\mu^{\delta_{f}} .
$$

The powers of $\mu$ absorb the engineering dimensions of the bare vertex functions. These are given by $\delta_{f}=f(\varepsilon / 2-1)+4-\varepsilon$. The renormalized couplings $g_{a b}$ defined by the relations (14), (15) depend on the scale parameter $\mu$. By their dependence on $g_{a b}$ also the renormalization $Z$-factors implicitly depend on $\mu$. This dependence is expressed by the RG functions defined by the following relations:

$$
\mu \frac{\mathrm{d}}{\mathrm{d} \mu} g_{a b}=\beta_{a b}\left(g_{a^{\prime} b^{\prime}}\right)
$$




$$
\begin{aligned}
\mu \frac{\mathrm{d}}{\mathrm{d} \mu} \ln Z_{\phi_{a}} & =\eta_{\phi_{a}}\left(g_{a a}\right), \\
\mu \frac{\mathrm{d}}{\mathrm{d} \mu} \ln Z_{* f_{1} f_{2}} & =\eta_{* f_{1} f_{2}}\left(g_{a b}\right), \\
\mu \frac{\mathrm{d}}{\mathrm{d} \mu} \ln Z_{\mathrm{MAW} f} & =\eta_{f}^{\mathrm{MAW}}\left(g_{a b}\right) .
\end{aligned}
$$

The function $\eta_{\phi_{a}}$ describes the pair correlation critical exponent, while the functions $\eta_{* f_{1} f_{2}}$ and $\eta_{f}^{\mathrm{MAW}}\left(g_{a b}\right)$ define the set of exponents for copolymer stars and the stars of mutually avoiding walks. Note that $Z_{* 20}$ renormalizes the vertex function with a $\phi^{2}$ insertion which coincides with $\Gamma^{(* 20)}$. Consequently, the usually defined correlation length critical exponent $\nu$ is expressed in terms of functions $\eta_{* 20}$ and $\eta_{\phi}$.

Expressions for the $\beta$ and $\eta$ functions will be discussed in the next sections together with a study of the RG flow and the fixed points of the theory.

\section{Homogeneous star polymer in three dimensions}

First we give numerical results for a homogeneous polymer star constituted by $f$ polymer chains of the same species. The scaling of a star is governed by the laws (4). For one species of polymers $(a, b=1)$, corresponding RG function (23)-(25) depend on a single coupling $g_{a b}=g$ and reduce to a single functions $\beta_{a b}\left(g_{a^{\prime} b^{\prime}}\right) \equiv \beta(g)$, $\eta_{\phi_{a}}\left(g_{a a}\right) \equiv \eta_{\phi}(g)$ and a set of functions $\eta_{* f_{1} f_{2}}\left(g_{a b}\right) \equiv \eta_{*\left(f_{1}+f_{2}\right)}(g)$ with $f_{1}+f_{2}=f$. Here, the function $\beta(g)$ is a standard $\beta$-function of the $O(m=0)$-symmetric theory. In the infrared-stable fixed point $g^{*}$ :

$$
\beta\left(g^{*}\right)=0,\left.\quad \frac{\mathrm{d} \beta(g)}{\mathrm{d} g}\right|_{g=g^{*}}>0 ;
$$

the function $\eta_{\phi}(g)$ gives the pair correlation function critical exponent $\eta$ :

$$
\eta=\eta\left(g^{*}\right)
$$

whereas the functions $\eta_{* f}(g)$ give the scaling exponents $\eta_{f}(4)$ :

$$
\eta_{f}=\eta_{* f}\left(g^{*}\right) .
$$

The RG functions (27)-(29) are obtained in the form of perturbation theory series. In the massive RG scheme, these are the series in a renormalized coupling $g$ for fixed space dimension $d$ whereas the zero-mass renormalization expansion is performed both in $g$ and in $\varepsilon=4-d$. Increasing the order of perturbation theory corresponds to increasing the number of loops when the expansion is written in terms of Feynman diagrams. That is why sometimes the expansions are classified in successive approximations in number of loops. The functions (27), (28) are the standard RG functions of the $O(m=0)$ symmetric theory. They are known by now in high orders of perturbation theory, whereas the functions (29) have been obtained only up to three loops. In the field theory they are related to the anomalous dimension of composite operators of traceless symmetry [15]. In polymer language the star exponents 
Table 1. Values of the star exponent $\gamma_{f}$ obtained in three-dimensional theory (columns 2,3,4,5) in comparison with the results of $\varepsilon$-expansion (columns 6,7,8) and Monte-Carlo simulations (column 9). See the text for a full description.

\begin{tabular}{|c|c|c|c|c|c|c|c|c|}
\hline \multirow[t]{2}{*}{$f$} & \multicolumn{4}{|c|}{ pseudo- $\varepsilon$ expansion $[22]$} & \multirow{2}{*}{\multicolumn{3}{|c|}{$\begin{array}{c}\varepsilon \text {-expansion } \\
{[8]}\end{array}$}} & \multirow{2}{*}{$\begin{array}{l}\mathrm{MC} \\
{[25,26]}\end{array}$} \\
\hline & Pade & Borel & conf.n & ipping & & & & \\
\hline 3 & 1.06 & 1.05 & 1.06 & 1.06 & 1.05 & 1.05 & 1.07 & 1.09 \\
\hline 4 & 0.86 & 0.86 & .86 & .83 & 0.84 & 0.83 & 0.85 & 0.88 \\
\hline 5 & 0.61 & 0.61 & .58 & .56 & 0.53 & 0.52 & 0.55 & 0.57 \\
\hline 6 & 0.32 & 0.32 & .24 & .22 & 0.14 & 0.18 & & 0.16 \\
\hline 7 & -0.02 & -0.01 & -.17 & -.17 & -0.33 & -0.20 & & \\
\hline 8 & -0.40 & -0.36 & -.63 & -.62 & -0.88 & -0.60 & & $(-0.99,-0.30)$ \\
\hline 9 & -0.80 & -0.72 & -1.14 & -1.11 & -1.51 & -1.01 & & \\
\hline
\end{tabular}

were originally calculated to order $\varepsilon$ in [20], the $\varepsilon^{2}$ results have been obtained in [7,21], whereas $\varepsilon^{3}$ expansions are given in [8]. The $\varepsilon$-expansion for the exponent $\eta_{f}$ reads $[15,8]$ :

$$
\eta_{f}=-\frac{\varepsilon}{8} f(f-1)\left[1-\frac{\varepsilon}{32}(8 f-25)+\frac{\varepsilon^{2}}{64}\left[(28 f-89) \zeta(3)+8 f^{2}-49 f+\frac{577}{8}\right]+O\left(\varepsilon^{3}\right)\right] .
$$

In the massive RG scheme, the three-loop expansions for the $d=3$ star exponents were obtained in the form of pseudo- $\varepsilon$ expansion in [22]. However, as already noted above, the perturbation theory expansions of the renormalized field theory have zero radius of convergence and are asymptotic at best. Therefore, in order to make numerical estimates for the expansions one should apply an appropriate resummation procedure. Here, we will not describe the different ways of resummation which were applied to the series for the star exponents referring rather to the papers $[8,9,22,24]$ where they are given in detail. For the purpose of the present review we just mention that a Borel transform was applied to get rid of the (conjectured) factorial divergence of the perturbation theory terms [17]. Subsequently, the expansion for the Borel transformed function was refined by a conformal mapping from the initial variable defined on the cut-plane to a disk keeping the origin invariant. Another scheme of resummation exploits the analytic continuation of the series for the Borel transformed function by means of Padé approximant (Padé-Borel resummation).

In table 1 we give the numerical results for the star exponents $\gamma_{f}$ as obtained by the fixed $d=3 \mathrm{RG}$ technique and in the $\varepsilon$-expansion in comparison with the data of the numerical simulation. First, we give the values of the exponent $\gamma_{f}$ obtained in the fixed $d=3$ technique by Padé-Borel resummation [22]: the second column contains the value of the $\gamma_{f}$ obtained directly from the resummation of the series for $\gamma_{f}$, while the third column gives the $\gamma_{f}$ based on the resummed series for the star exponents $x_{f}^{\prime}$. These are connected with the $\gamma_{f}$ via the scaling relation:

$$
\gamma_{f}=1-\nu x_{f}^{\prime}+[\nu(2-\eta / 2)-1] f
$$


substituting the well-known values of the exponents $\nu(d=3)=0.588, \eta(d=3)=$ 0.027. Columns 4 and 5 give $\gamma_{f}$ obtained by the resummation using the conformal mapping technique [22]: the resummation of the series $\gamma_{f}(\tau)$ (fourth column) and the resummation of the series $x_{f}^{\prime}(\tau)$ (fifth column). The next columns show the results obtained by the $\varepsilon^{3}$-expansion using: simple Padè approximation (the 6th column) and Padè-Borel analysis neglecting or exploiting the exact results for $d=2$ (the 7th and 8th columns, respectively) [8]. The last column contains Monte-Carlo data $[25,26]$. For low numbers of arms $F \leqslant 5$ the results of the different approaches agree reasonably well and are also close to the values obtained by $\mathrm{MC}$ simulation.

The data collected in table 1 was obtained using two different renormalization schemes as well as different procedures for the resummation of the resulting asymptotic series. Thus, table 1 gives a test for the stability of the results under the changes of the calculational scheme. Obviously, for higher numbers $f>5$ of arms, the coincidence of the results is no longer good. The main reason for this is that calculating the exponents, combinatorial factors lead to an expansion in $f \varepsilon$ for the $\varepsilon$ expansion and of $f g$ when directly expanding in a renormalized coupling $g$. For such large values of the expansion parameter, even resummation of the series fails. For larger numbers of arms, other approaches to the theory of polymer stars, like a self consistent field approximation, might be more useful.

\section{Copolymers and copolymer stars}

Now let us pass to the case of a polymer star constituted by two species of polymers. It is described by the Lagrangian (10) with interactions $u_{11}, u_{22}$ between the polymers of the same species and $u_{12}=u_{21}$ between the polymers of different species. Such a Lagrangian is used to describe the system of polymers of two species immersed in a solvent: the so-called ternary solution [27]. A comprehensive analysis of the fixed point behaviour of a ternary solution was given in [14] where the RG flow of the theory was calculated within the massless renormalization and is known by now to the third loop order of the $\varepsilon$-expansion. Note, that for the diagonal coupling $g_{a a}$ the corresponding expressions are also found in the polymer limit $m=0$ of the $O(m)$-symmetric $\phi^{4}$ model. They are known in even higher orders of perturbation theory. In the massive RG approach, the corresponding expressions were obtained in [9]. The equations for the fixed points (FP) $\mathrm{P}\left(\left\{g_{11}^{*}, g_{22}^{*}, g_{12}^{*}\right\}\right)$ of the $\beta$-functions read:

$$
\begin{aligned}
\beta_{g_{a a}}\left(g_{a a}^{*}\right) & =0, \quad a=1,2, \\
\beta_{g_{12}}\left(g_{11}^{*}, g_{22}^{*}, g_{12}^{*}\right) & =0 .
\end{aligned}
$$

As is well known, the first equation has two solutions $g_{a a}^{*}=0, g_{\mathrm{S}}^{*}$. For the second equation, one finds a total of 8 FPs depending on the choice for $g_{a a}^{*}$. The trivial FPs are $\mathrm{G}_{0}(0,0,0), \mathrm{U}_{0}\left(g_{\mathrm{S}}^{*}, 0,0\right), \mathrm{U}_{0}^{\prime}\left(0, g_{\mathrm{S}}^{*}, 0\right), \mathrm{S}_{0}\left(g_{\mathrm{S}}^{*}, g_{\mathrm{S}}^{*}, 0\right)$, all corresponding to $g_{12}^{*}=0$. The non-trivial FPs are found as $\mathrm{G}\left(0,0, g_{\mathrm{G}}^{*}\right), \mathrm{U}\left(g_{\mathrm{S}}^{*}, 0, g_{\mathrm{U}}^{*}\right), \mathrm{U}^{\prime}\left(0, g_{\mathrm{S}}^{*}, g_{\mathrm{U}}^{*}\right)$, and $\mathrm{S}\left(g_{\mathrm{S}}^{*}, g_{\mathrm{S}}^{*}, g_{\mathrm{S}}^{*}\right)$. In the three dimensional space of couplings $g_{11}, g_{22}, g_{12}$ these FPs are placed at the corners of a cube that is deformed in the $g_{12}$ direction (see figure 1 ). 
Looking for the stability of the above described fixed points one finds that only the fixed point $S$ is stable [14]. In the excluded volume limit of infinitely long chains, the behaviour of a system of two polymer species is thus described by the same scaling laws as a solution of only one polymer species. Nevertheless, taking into account that real polymer chains are not infinitely long, one may also find crossover phenomena which are governed by the unstable fixed points. Knowing the complete RG flow, allows one to describe crossover phenomena in the whole accessible region [14]. However, for the purpose of our review we are interested only in the values of the fixed points and in the properties of the star vertex functions at these points.

Let us define the asymptotic values of the copolymer star exponents and the mutually avoiding walk (MAW) star exponents by:

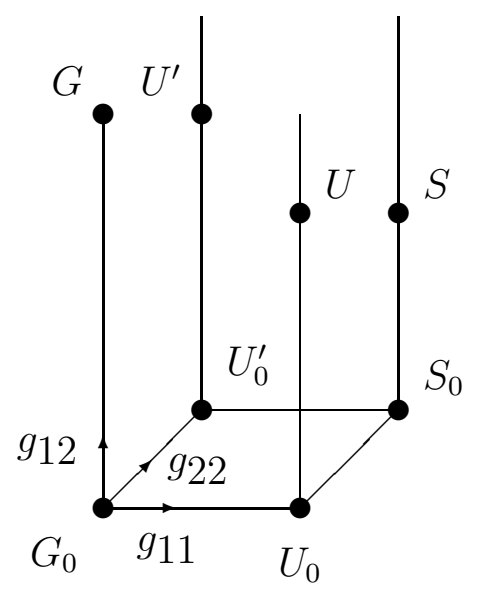

Figure 1. Fixed points (FPs) of ternary polymer solution. The trivial FPs $G_{0}$, $U_{0}, U_{0}^{\prime}, S_{0}$ correspond to a vanishing mutual interaction. The non-trivial FPs $G$, $U, U^{\prime}, S$ correspond to a non-vanishing mutual interaction $\left(g_{12} \neq 0\right)$.

$$
\begin{aligned}
\eta_{f_{1} f_{2}}^{\mathrm{S}} & =\left.\eta_{* f_{1} f_{2}}\left(g_{a b}\right)\right|_{S}, \\
\eta_{f_{1} f_{2}}^{\mathrm{G}} & =\left.\eta_{* f_{1} f_{2}}\left(g_{a b}\right)\right|_{G}, \\
\eta_{f_{1} f_{2}}^{\mathrm{U}} & =\left.\eta_{* f_{1} f_{2}}\left(g_{a b}\right)\right|_{U}=\left.\eta_{* f_{2} f_{1}}\left(g_{a b}\right)\right|_{U^{\prime}}, \\
\eta_{f}^{\mathrm{MAW}} & =\left.\eta_{f}^{\mathrm{MAW}}\left(g_{a b}\right)\right|_{G} .
\end{aligned}
$$

Taking into account the nature of the fixed points, where the exponents (32)-(36) are defined, one arrives at the following physical interpretation:

- the exponent $\eta_{f_{1} f_{2}}^{\mathrm{G}}$ describes a star of $f_{1}$ random walks of the 1 st type and $f_{2}$ random walks of the 2nd type all of which are not selfavoiding; only the chains of the 1st type avoid those of the 2nd type and vice versa;

- the exponent $\eta_{f_{1} f_{2}}^{\mathrm{U}}$ describes a star of $f_{1}$ self and mutually avoiding polymer chains walks of the 1 st type and $f_{2}$ random walks which only avoid those of the 1st type;

- the exponent $\eta_{f_{1} f_{2}}^{\mathrm{MAW}}$ describes a star of $f_{1}+f_{2}$ random walks all of which are not selfavoiding; however, each chain avoids any other chain of the star;

- exponent $\eta_{f_{1} f_{2}}^{\mathrm{S}}$ describes a star of $f_{1}+f_{2}$ self and mutually avoiding chains. The exponent can also be expressed by $\eta_{f_{1} f_{2}}^{\mathrm{S}}=\eta_{f_{1}+f_{2}, 0}^{\mathrm{U}}$ (This relation is broken 
for the exact results known in $d=2$ where a finite effect remains in the zero walk limit). This situation has already been discussed in the previous section.

Starting from the expressions for the fixed points (available both in the form of a three-loop $\varepsilon$ expansion [14] or in the form of pseudo- $\varepsilon$ expansion at fixed $d$ [9]) and the relations (33)-(36), one can find the series for the star exponents. In the $\varepsilon$-expansion the following expansions for $\eta_{f_{1} f_{2}}$ are obtained [9]:

$$
\begin{aligned}
\eta_{f_{1} f_{2}}^{\mathrm{G}}(\varepsilon)= & -f_{1} f_{2} \frac{\varepsilon}{2}+f_{1} f_{2}\left(f_{2}-3+f_{1}\right) \frac{\varepsilon^{2}}{8} \\
& -f_{1} f_{2}\left(f_{2}-3+f_{1}\right)\left(f_{1}+f_{2}+3 \zeta(3)-3\right) \frac{\varepsilon^{3}}{16}, \\
\eta_{f_{1} f_{2}}^{\mathrm{U}}(\varepsilon)= & f_{1}\left(1-f_{1}-3 f_{2}\right) \frac{\varepsilon}{8} \\
+ & f_{1}\left(25-33 f_{1}+8{f_{1}}^{2}-91 f_{2}+42 f_{1} f_{2}+18 f_{2}{ }^{2}\right) \frac{\varepsilon^{2}}{256} \\
+ & f_{1}\left(577-969 f_{1}+456 f_{1}{ }^{2}-64 f_{1}{ }^{3}-2463 f_{2}+2290 f_{1} f_{2}\right. \\
& -492 f_{1}{ }^{2} f_{2}+1050 f_{2}{ }^{2}-504 f_{1} f_{2}{ }^{2}-108 f_{2}{ }^{3}-712 \zeta(3)+936 f_{1} \zeta(3) \\
& \left.-224 f_{1}{ }^{2} \zeta(3)+2652 f_{2} \zeta(3)-1188 f_{1} f_{2} \zeta(3)-540 f_{2}{ }^{2} \zeta(3)\right) \frac{\varepsilon^{3}}{4096}, \\
\eta_{f_{1} f_{2}}^{\mathrm{MAW}}(\varepsilon)= & -\left(f_{1}-1\right) f_{1} \frac{\varepsilon}{4}+f_{1}\left(f_{1}-1\right)\left(2 f_{1}-5\right) \frac{\varepsilon^{2}}{16} \\
& -\left(f_{1}-1\right) f_{1}\left(4 f_{1}{ }^{2}-20 f_{1}+8 f_{1} \zeta(3)-19 \zeta(3)+25\right) \frac{\varepsilon^{3}}{32} .
\end{aligned}
$$

Here $\zeta(3) \simeq 1.202$ is the Riemann $\zeta$-function. The above formulas reproduce the 3rd order calculations [8] of the scaling exponents of homogeneous polymer stars $\eta_{f}=\eta_{f, 0}^{\mathrm{U}}$ given in formula (35) of the previous section. The corresponding pseudo- $\varepsilon$ expansion for the exponents $\eta_{f_{1} f_{2}}$ obtained in the massive scheme may be found in [9]. We do not give it here explicitly although in what follows below we will give numerical values of the exponents obtained in both approaches. It has been pointed out in [28] that for the exponent $\eta_{12}^{\mathrm{G}}$ an exact estimate equal to our first order contribution may be found. It is indeed remarkable that all higher order contributions to $\eta_{12}^{\mathrm{G}}$ vanish in both approaches, $\eta_{12}^{\mathrm{G}}=-\varepsilon$ being an exact result [23].

With these exponents one can describe the scaling behaviour of polymer stars and networks of two components, generalizing the relation for single component networks [7]. In the notation of (4) one finds for the number of configurations of a network $\mathcal{G}$ of $F_{1}$ and $F_{2}$ chains of species 1 and 2

$$
\mathcal{Z}_{\mathcal{G}} \sim(R / \ell)^{\eta_{\mathcal{G}}-F_{1} \eta_{20}-F_{2} \eta_{02}}, \quad \text { with } \eta_{\mathcal{G}}=-\mathrm{d} L+\sum_{f_{1}+f_{2} \geqslant 1} N_{f_{1} f_{2}} \eta_{f_{1} f_{2}}
$$

where $L$ is the number of loops and $N_{f_{1} f_{2}}$ is the number of vertices with $f_{1}$ and $f_{2}$ arms of species 1 and 2 in the network $\mathcal{G}$. To receive an appropriate scaling law we 
Table 2. Values of the copolymer star exponent $\eta_{f_{1} f_{2}}^{\mathrm{G}}$ for $d=3$ obtained by the $\varepsilon$-expansion $(\varepsilon)$ and by fixed dimension technique $(3 d)$.

\begin{tabular}{|r|rr|rr|rr|rr|rr|rr|}
\hline$f_{1}$ & \multicolumn{2}{|c|}{1} & \multicolumn{2}{|c|}{2} & \multicolumn{2}{|c|}{3} & \multicolumn{2}{|c|}{5} & \multicolumn{2}{|c|}{6} \\
$f_{2}$ & $\varepsilon$ & $3 d$ & $\varepsilon$ & $3 d$ & $\varepsilon$ & $3 d$ & $\varepsilon$ & $3 d$ & $\varepsilon$ & $3 d$ & $\varepsilon$ & $3 d$ \\
\hline 1 & -0.56 & -0.58 & -1.00 & -1.00 & -1.33 & -1.35 & -1.63 & -1.69 & -1.88 & -1.98 & -2.10 & -2.24 \\
2 & & & -1.77 & -1.81 & -2.45 & -2.53 & -3.01 & -3.17 & -3.51 & -3.75 & -3.95 & -4.28 \\
3 & & & & & -3.38 & -3.57 & -4.21 & -4.50 & -4.94 & -5.36 & -5.62 & -6.15 \\
4 & & & & & & & -5.27 & -5.71 & -6.24 & -6.84 & -7.12 & -7.90 \\
5 & & & & & & & & & -7.42 & -8.24 & -8.50 & -9.54 \\
6 & & & & & & & & & & & -9.78 & -11.07 \\
\hline
\end{tabular}

Table 3. The values of the copolymer star exponent $\eta_{f_{1} f_{2}}^{\mathrm{U}}$ at $d=3$ obtained by the $\varepsilon$-expansion $(\varepsilon)$ and by fixed dimension technique $(3 d)$.

\begin{tabular}{|r|rr|rr|rr|rr|rr|rr|}
\hline$f_{1}$ & \multicolumn{2}{|c|}{1} & 2 & \multicolumn{2}{|c|}{3} & 4 & \multicolumn{2}{|c|}{5} & \multicolumn{2}{|c|}{6} \\
$f_{2}$ & $\varepsilon$ & $3 d$ & $\varepsilon$ & $3 d$ & $\varepsilon$ & $3 d$ & $\varepsilon$ & $3 d$ & $\varepsilon$ & $3 d$ & $\varepsilon$ & $3 d$ \\
\hline 0 & 0 & 0 & -0.28 & -0.28 & -0.75 & -0.76 & -1.36 & -1.38 & -2.07 & -2.14 & -2.88 & -3.01 \\
1 & -0.43 & -0.45 & -0.98 & -0.98 & -1.64 & -1.67 & -2.39 & -2.47 & -3.21 & -3.38 & -4.11 & -4.40 \\
2 & -0.79 & -0.81 & -1.58 & -1.60 & -2.44 & -2.52 & -3.33 & -3.50 & -4.28 & -4.57 & -5.29 & -5.73 \\
3 & -1.09 & -1.09 & -2.13 & -2.19 & -3.16 & -3.30 & -4.20 & -4.48 & -5.28 & -5.71 & -6.41 & -7.03 \\
4 & -1.35 & -1.37 & -2.61 & -2.71 & -3.82 & -4.04 & -5.02 & -5.40 & -6.24 & -6.81 & -7.48 & -8.28 \\
5 & -1.60 & -1.64 & -3.05 & -3.21 & -4.44 & -4.75 & -5.80 & -6.30 & -7.15 & -7.89 & -8.51 & -9.50 \\
6 & -1.81 & -1.89 & -3.46 & -3.68 & -5.01 & -5.42 & -6.53 & -7.15 & -8.02 & -8.92 & -9.50 & -10.69 \\
\hline
\end{tabular}

assume the network to be built of chains which for both species will have the same coil radius $R$ when isolated.

In order to calculate numerical values for the exponents $\eta_{f_{1} f_{2}}^{\mathrm{G}}, \eta_{f_{1} f_{2}}^{\mathrm{U}}$ and $\eta_{f}^{\mathrm{MAW}}$ in [9,29-31] the Borel resummation refined by conformal mapping was applied to the series (37)-(39) as well as to the appropriate series in pseudo- $\varepsilon$ expansion. The resummation showed that the two schemes yield consistent numerical estimates. Our tables 2, 3, and 4 list the results.

Comparing the numerical values listed in the above tables it is convincing that the two approaches and the different resummation procedures all lead to the results which lie within a bandwidth of consistency, which is broadening for larger values of number of chains $f_{1}, f_{2}>1$. This is not surprising. One can see, e.g. from the formulas (37)-(39), that the expansion parameters are multiplied by $f_{1}$ and $f_{2}$. Rather, it is remarkable that even for a total number of chains of the order of 10 (see tables 2,3) one still receives the results which are comparable to each other.

In the next three sections we will briefly review several phenomena where star exponents become important. 
Table 4. The values of the $\eta_{f}^{\mathrm{MAW}}$ exponents of stars of mutually avoiding walks for $d=3, d=2$ obtained by the $\varepsilon$-expansion $(\varepsilon)$ and by the fixed dimension technique $(3 d, 2 d)$. The last column gives the exact conjecture for $d=2[16]$.

\begin{tabular}{|l|ll|lll|}
\hline & \multicolumn{2}{|c|}{$d=3$} & \multicolumn{3}{c|}{$d=2$} \\
$f$ & $\varepsilon$ & $3 d$ & $\varepsilon$ & $2 d$ & exact \\
\hline 1 & 0 & 0 & 0 & 0 & -.250 \\
2 & -.56 & -.56 & -1.20 & -1.19 & -1.250 \\
3 & -1.38 & -1.36 & -2.71 & -2.60 & $-2.916(6)$ \\
4 & -2.36 & -2.34 & -4.36 & -4.07 & -5.250 \\
5 & -3.43 & -3.43 & -6.04 & -5.61 & -8.250 \\
6 & -4.58 & -4.64 & -7.78 & -7.17 & $-11.916(6)$ \\
\hline
\end{tabular}

\section{Colloids with polymer stars: The interaction}

Let us now consider the effective interaction between the cores of two star polymers at a small distance (small on the scale of the size $R_{g}$ of the star). Let us analyse the general case of two stars of different functionalities $f_{1}$ and $f_{2}$. The power law for the partition sum $\mathcal{Z}_{f_{1} f_{2}}^{(2)}$ of such two star polymers at a distance $r$ [7],

$$
\mathcal{Z}_{f_{1} f_{2}}^{(2)}(r) \sim r^{\Theta_{f_{1} f_{2}}^{(2)}}
$$

is governed by the contact exponent $\Theta_{f_{1} f_{2}}^{(2)}$. As we have seen in the section 2 in the formalism of the $m=0$ component model, the core of a star polymer corresponds to a local product of $f$ spin fields $\phi_{1}(\mathbf{x}) \cdots \phi_{f}(\mathbf{x})$, each representing the endpoint of one polymer chain. The probability of approach of the two cores of the star polymers at a small distance $r$ in these terms is described by a short distance expansion for the composite fields. The short distance expansion provides the scaling relations between the exponents $\Theta_{f_{1} f_{2}}$ and the star exponents $\gamma_{f}\left(\right.$ or $\eta_{f}$ ):

$$
\begin{aligned}
\nu \Theta_{f_{1} f_{2}} & =\left(\gamma_{f_{1}}-1\right)+\left(\gamma_{f_{2}}-1\right)-\left(\gamma_{f_{1}+f_{2}}-1\right), \\
\Theta_{f_{1} f_{2}} & =\eta_{f_{1}}+\eta_{f_{2}}-\eta_{f_{1}+f_{2}} .
\end{aligned}
$$

The mean force $F_{f_{1} f_{2}}^{(2)}(r)$ between two star polymers at a short distance $r$ is easily derived from the effective potential $V_{f_{1} f_{2}}^{\text {eff }}(r)=\log \mathcal{Z}_{f_{1} f_{2}}^{(2)}(r)$ as

$$
F_{f_{1} f_{2}}^{(2)}(r)=\frac{\Theta_{f_{1} f_{2}}^{(2)}}{r}, \text { with } \Theta_{f f}^{(2)} \approx \frac{5}{18} f^{3 / 2} .
$$

The factor 5/18 is found by matching the cone approximation for $\Theta_{f f}^{(2)}$ (see formula (5)) to the known values of the contact exponents for $f=1,2$ [34]. This matching, in turn, proposes an approximate value for the $\eta_{f}$ exponents,

$$
\eta_{f} \approx-\frac{5}{18}\left(2^{3 / 2}-2\right) f^{3 / 2}-f \eta_{1}
$$


Table 5. The prefactor $\Theta_{f_{1} f_{2}}$ of the force between two star polymers at a short distance calculated in non-resummed 1-loop and resummed 3-loop RG analysis in comparison to the result of the cone approximation.

\begin{tabular}{|l|r|r|r|r|r|l|}
\hline$\Theta_{f_{1} f_{2}}$ & $f_{2}=1$ & $f_{2}=2$ & $f_{2}=3$ & $f_{2}=4$ & $f_{2}=5$ & app. \\
\hline$f_{1}=1$ & -.21 & -.42 & -.63 & -.85 & -1.06 & 1-loop \\
$f_{1}=1$ & -.28 & -.48 & -.62 & -.76 & -.87 & 3-loop \\
$f_{1}=1$ & -.27 & -.45 & -.60 & -.73 & -.84 & cone \\
& & & & & & \\
$f_{1}=2$ & & -.85 & -1.27 & -1.70 & & 1-loop \\
$f_{1}=2$ & & -.82 & -1.10 & -1.35 & & 3-loop \\
$f_{1}=2$ & & -.78 & -1.05 & -1.29 & & cone \\
& & & & & & \\
$f_{1}=3$ & & & -1.91 & & & 1-loop \\
$f_{1}=3$ & & & -1.49 & & & 3-loop \\
$f_{1}=3$ & & & -1.44 & & & cone \\
\hline
\end{tabular}

where $-f \eta_{1}$ is introduced for consistency with the exact result $\eta_{1}=0$. This assumption nicely reproduces the contact exponents as derived from 3-loop perturbation theory [9] as is displayed in table 5 taken from the [33].

In table 5 we use the approximate values of $\eta_{f}$ to calculate the cone estimation of the contact exponents and compare these with the corresponding values of a renormalization group calculation. A perturbation series is taken in terms of an $\varepsilon$ expansion. The result labelled '1-loop' corresponds to optimal truncation of the series by simply inserting $\varepsilon=1$ or $d=3$ in the first order term of the expansion. The 3 loop result includes a resummation procedure that takes into account the asymptotic nature of the series [9]. The large $f$ result corresponds to the cone approximation (5).

The above data convincingly shows that the large $f$ approximation for the short distance force between two star polymers can be consistently fitted to the results of perturbation theory for low values of the functionality $f$ of the stars. Note, that increasing the number of chains $f$, the polymer stars interpolate between the properties of linear polymers and polymeric micelles [35]. This approach is general enough to describe the interaction between two stars of different functionalities $f_{1}$ and $f_{2}$. This is essential in extending the theory of colloidal solutions of star polymers to general polydispersity in $f$ as it appears naturally in any real experiment.

\section{Brownian motion near an absorbing polymer star}

Now, let us turn to another physical phenomenon that is described in terms of the star exponents. To this end we consider a model proposed by Cates and Witten [28] to describe the flow of diffusing particles near an absorbing object, which may be a polymer chain ( = a selfavoiding walk (SAW)) or a random walk (RW). The flux of particles on the absorbing chain is defined by the density of incoming 
particles close to the absorber (see figure 2). Taken $\rho(r)$ to be the probability density of incoming particles, the problem is to solve the steady-state diffusion equation (Laplace equation)

$$
\nabla^{2} \rho(r)=0
$$

with boundary conditions for a given absorbing polymer

$$
\begin{aligned}
& \rho(r)=0 \quad \text { on the surface of the absorber, } \\
& \rho(r)=\rho_{\infty}=\text { const for } \quad|r| \rightarrow \infty \text {. }
\end{aligned}
$$

One of the motivations for introducing this model was to gain insight for the problem of diffusion limited aggregation (DLA) [39]. The latter phenomenon is much more complicated because of the fact that for DLA the boundary conditions are given on the surface of the growing aggregate itself. The process described by equations (45), (46) may be rather considered as a diffusion limited catalysis, when particles of one type interact with a prescribed fractal (a catalyzing polymer) and transform into the other type [38] (see section 7 for more details).

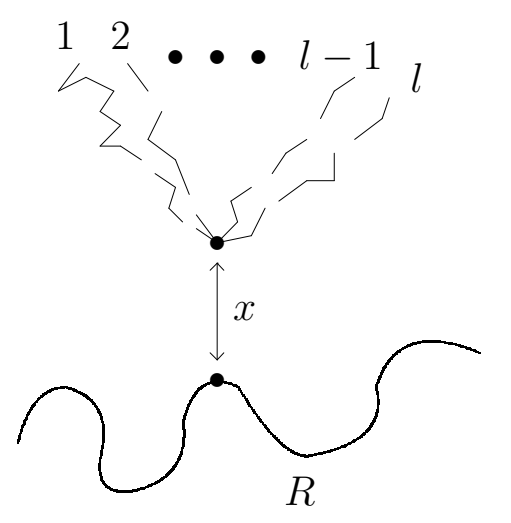

Figure 2. Star of $l$ random walks at distance $x$ from an absorbing polymer of size $R$.
Let the absorbing polymer of size $R$ be chosen from the well defined ensemble of SAWs. Then one may introduce exponents that govern the scaling of the moments of the field $\rho(r)$ close to the surface of the absorber (see figure 2). For distances $R \gg r>a$ ( $a$ being a cut off) the averaged moments $\left\langle\rho(r)^{n}\right\rangle$ are expected to scale as [28]

$$
\left\langle\rho(r)^{n}\right\rangle \sim(R / r)^{-\lambda(n)}
$$

where $\langle\ldots\rangle$ denotes the average over the ensemble of polymers. Taking that the flux $\phi(x)$ onto any randomly chosen point $x$ of the absorber is proportional to the field $\rho(r)$ at a point that is as close as a cut off length $a$ from the absorber, one finds for the averaged moments of the flux:

$$
\left\langle\phi^{n}\right\rangle \sim(R / a)^{-\lambda(n)}
$$

There is a natural way to associate the exponents $\lambda(n)(47)$, (48) with the copolymer star exponents $\eta_{f_{1} f_{2}}(34)$, (35). The central idea that allows one to calculate the properties of the solutions of equation (45) with the boundary conditions (46) is that a path integral representation both for the field $\rho(r)$ and for the absorber (being a RW or a SAW) is possible $[28,38]$. In terms of the path integral solution of the Laplace equation, one finds that $\rho(r)$ at point $r$ near the absorber is proportional to the number of RW that end at point $r$ and avoid the absorber. The $n$th power of this field is proportional to the $n$th power of the above mentioned number, i.e. it is 
defined by the partition function of a star polymer with $n$ arms (12) (the latter is shown by dashed lines in figure 2). Furthermore, introducing the mutual avoidance conditions between the " $n$-arm star" and the 2 -arm polymer (representing the absorber), one has to calculate the partition function of a co-polymer star consisting of chains of two different species that avoid each other. These correspond to the trajectories of diffusing particles (being RW) and the absorbing polymer (which is chosen to be a RW or SAW). Making use of the theory of copolymer stars and networks [9], one may relate [24] the spectrum of exponents $(47)$, (48) to the exponents that define the scaling properties of co-polymer stars. In particular, as we have seen in the section 4 for a co-polymer star consisting of $f_{1}$ chains of species 1 and $f_{2}$ chains of species 2 , the scaling of the number of configurations $Z_{*}$ is governed by the exponents $\eta_{f_{1} f_{2}}(40)$. By means of a short-chain expansion [36] it is clear that only the (smaller) length scale of the absorbing polymer remains and the set of exponents $\eta_{f_{1} f_{2}}$ can be related to the exponents $\lambda(n)$ (47) that govern the scaling of the $n$th moment of the flux onto an absorbing linear chain. Considering the absorber to be either a RW or a SAW the exponents read:

$$
\begin{aligned}
\lambda_{\mathrm{RW}}^{\mathrm{G}}(n) & =-\eta_{2 n}^{\mathrm{G}}, \\
\lambda_{\mathrm{SAW}}^{\mathrm{U}}(n) & =-\eta_{2 n}^{\mathrm{U}}+\eta_{20} .
\end{aligned}
$$

Numerical values for the exponents $\lambda(n)$ may be easily extracted from the numerical values of the exponents $\eta_{2 n}$ given in tables 2,3 . Moreover, one can consider an absorber in the form of an $f_{1}$-arm star and generalize the above considerations looking for the scaling laws of diffusing particles in the vicinity of the core of such a polymer star. Again, the scaling may be expressed in terms of the co-polymer star exponents [24].

It is well known that polymer chains are fractals with a fractal dimension defined by a correlation length exponent: $d_{f}=1 / \nu$. It appears that the behaviour of Brownian motion in the vicinity of an absorbing star polymer possesses multifractal [37] features. These are analyzed in detail in [24,30].

\section{Diffusion-controlled reactions in presence of polymers}

As mentioned in the preceding section, one of the processes described by equations (45), (46) may also be considered as diffusion limited catalysis. In this section, we will comment on a general phenomenon which can again be described in terms of the star exponents: chemical reactions between diffusing reactants. Examples of such reactions can be found in different systems, ranging from biological systems to nuclear reactors (see e.g. [40] and references therein). One more place where these reactions appear as a limiting stage is the aggregation models [41]. Of particular interest are the reactions between reactants of different nature: particles $A$ which freely diffuse in a solution and particles $B$ which are attached to polymer chains immersed in the same solvent [38] (the concentration of polymers being low enough to allow to neglect the inter-chain interaction). Such a process may be also considered 
as a trapping reaction of $n$ particles of $A$ type and traps $B$ :

$$
A^{n}+B \rightarrow B \text {. }
$$

The reaction rate $k_{n}$ of (51) in the vicinity of a certain trap on the polymer of size $R$ is proportional to the averaged moments of the concentration $\rho$ of diffusing particles near this trap. From (47) we get that the reaction rate scales with $R$ as [28]:

$$
k_{n} \sim\left\langle\rho^{n}\right\rangle \sim(R / l)^{-\lambda_{n}},
$$

with $l$ being a characteristic length scale.

The scaling exponents $\lambda_{n}$ (52) were considered in the previous section (see formulas (49) and (50)). Now, considering the absorber to be either a RW star or a SAW star of $m$ chains, we define the exponents $\lambda_{m n}$ which can be related to the familiar copolymer star exponents $\eta_{m n}$ (35) via scaling laws:

$$
\begin{aligned}
\lambda_{m n}^{\mathrm{RW}} & =-\eta_{m n}^{\mathrm{G}}, \\
\lambda_{m n}^{\mathrm{SAW}} & =-\eta_{m n}^{\mathrm{U}}+\eta_{m 0}^{\mathrm{U}} .
\end{aligned}
$$

Let us note that the case $m=2$ corresponds to a trap located on the chain polymer, whereas $m=1$ corresponds to a trap attached at the polymer extremity.

Numerical values for the exponent $\lambda_{m n}$ as well as perturbation theory expansions for them follow from tables 2,3 and are analyzed in detail in [9,42-44]. Here, let us analyze several particular cases for an absorbing $m$-star with a reactant trap at its core and simply absorbing traps along all chains:

- For a given m-star absorber of size $R$, the reaction rate (52) scales as $k_{m n} \sim$ $(R / l)^{-\lambda_{m n}}$. The increase of the size $R$ by a factor of $a$ results in $k_{m n}^{\prime} \sim$ $(a R / l)^{-\lambda_{m n}}$ leading to:

$$
k_{m n}^{\prime} / k_{m n} \sim a^{-\lambda_{m n}}
$$

with $\lambda_{m n}$ positive: increasing $R$ by a factor of $a$ the reaction rate decreases $a^{-\lambda_{m n}}$ times due to the increase of absorbing traps.

- For a given reaction type (51) (i.e. for a fixed number $n$ of particles which are trapped simultaneously) attaching $m_{1}$ additional arms to an $m$-arm star absorber results in a decrease of the reaction rate:

$$
k_{\left(m+m_{1}\right), n} / k_{m n} \sim(R / l)^{-\left(\lambda_{\left(m+m_{1}\right), n}-\lambda_{m n}\right)},
$$

as far as $\lambda_{m_{2} n}>\lambda_{m_{1} n}$ for $m_{2}>m_{1}$.

- For a given m-star absorber the change of the type of reaction (51) to $A^{n_{1}}+$ $B \rightarrow B, n_{1}>n$ results in a decrease of the reaction rate:

$$
k_{m,\left(n+n_{1}\right)} / k_{m n} \sim(R / l)^{-\left(\lambda_{m,\left(n+n_{1}\right)}-\lambda_{m n}\right)},
$$

as far as $\lambda_{m n_{2}}>\lambda_{m n_{1}}$ for $n_{2}>n_{1}$; now, more particles need to find the trap at the core simultaneously for a reaction to take place. 


\section{Conclusions and outlook}

The notion of critical exponents is one of the central notions in the theory of critical phenomena. The precise determination of the set of critical exponents governing an equilibrium thermodynamic second order phase transitions provides a challenge in numerous studies. The exponents usually govern the power-law temperature behaviour which manifests itself in the vicinity of the critical point. In polymer theory, the direct "relatives" of the exponents governing temperature behaviour at the 2 nd order phase transitions are the exponents describing the configurational properties of long flexible polymer chains. They govern the scaling laws appearing in the limit of infinite chain length. These exponents are important in defining the entropy of a polymer solution, the radius of gyration of a polymer coil, the osmotic pressure of the polymer component in a solution, its rheologic properties, etc $[1,3]$. Both the 2nd order phase transition critical exponents and the polymer chain scaling exponents are closely related to each other and, finally, correspond to the second derivative of the appropriate free energy. However, in polymer theory there exist sets of exponents which do not have direct "relatives" in the theory of 2nd order phase transitions. These exponents describe the scaling of star polymers and polymer networks [7]. Whereas the scaling exponents for chain polymers are calculated by now in different techniques with the standard accuracy of modern theory of critical phenomena, this is not the case for the star exponents.

In the present paper we have reviewed some recent calculations of the critical exponents governing the scaling behaviour of star polymers. To calculate the critical exponents it is today standard to rely on the renormalization group technique improved by a resummation of asymptotic series [2,3]. The numbers provided in tables 1-4 of the paper provide reliable data which may be used in different problems where the star exponents become important. Some of the problems were mentioned in the sections 5-7: the interaction between star polymers in a good solvent, the Brownian motion near absorbing polymers, diffusion-controlled reactions in the presence of polymers.

\section{References}

1. see e.g.: de Gennes P.-G. Scaling Concepts in Polymer Physics. Ithaca and London, Cornell University Press, 1979.

2. Zinn-Justin J. Quantum Field Theory and Critical Phenomena. Oxford University Press, 1996; Kleinert H., Schulte-Frohlinde V. Critical Properties of $\phi^{4}$-Theories. Singapore, World Scientific, 2001.

3. des Cloizeaux J., Jannink G. Polymers in Solution. Oxford, Clarendon Press, 1990; Schäfer L. Excluded Volume Effects in Polymer Solutions. Berlin, Springer, 1999.

4. Guida R., Zinn Justin J. // J. Phys. A, 1998, vol. 31, p. 8104.

5. Nienhuis B. // Phys. Rev. Lett., 1982, vol. 49, p. 1062.

6. We neither give here examples of star polymers nor emphasize their importance in applications: rather we refer to the bulk of papers constituting this special issue of Condens. Matter Phys. and devoted to the subject. 
7. Duplantier B. // J. Stat. Phys., 1989, vol. 54, p. 581-680.

8. Schäfer L., von Ferber C., Lehr U., Duplantier B. // Nuc. Phys., 1992, vol. 374, p. 473-495.

9. von Ferber C., Holovatch Yu. // Phys. Rev. E, 1997, vol. 56, p. 6370-6386.

10. Duplantier B. // Phys. Rev. Lett., 1986, vol. 57, p. 941-945.

11. Duplantier B. // Phys. Rev. Lett., 1999, vol. 82, p. 880; Physica A, 1999, vol. 263, p. 452.

12. von Ferber C., Holovatch Yu. // Phys. Rev. E, 2002 (in print).

13. Ohno K., Binder K. // J. Phys. France, 1988, vol. 49, p. 1329.

14. Schäfer L., Lehr U., Kappeler C. // J. Phys. Paris I, 1991, vol. 1, p. 211-233.

15. Wallace D.J., Zia R.K.P. // J. Phys. C, 1975, vol. 8, p. 839.

16. Duplantier B. // Commun. Math. Phys., 1988, vol. 117, p. 279; Duplantier B., Kwon K.-H. // Phys. Rev. Lett., 1988, vol. 61, p. 2514-2517.

17. Hardy G. Divergent Series. Oxford University Press, 1948.

18. Wilson K.G., Fisher M.E. // Phys. Rev. Lett., 1972, vol. 28, p. 240.

19. Parisi G. // 1973 (unpublished); J. Stat. Phys., 1980, vol. 23, p. 49.

20. Miyake A., Freed K.F. // Macromolecules, 1983, vol. 16, p. 1228-1241.

21. Ohno K. // Phys. Rev. A, 1989, vol. 40, p. 1524-1538.

22. von Ferber C., Holovatch Yu. // Condens. Matter Phys., 1995, No. 5, p. 8-22;

Theor. Math. Phys. (Moscow), 1996, vol. 109, p. 34-50 (Engl. ed.: p. 1274-1286).

23. Semenov A.N. // J. Phys. France, 1988, vol. 49, p. 1353.

24. von Ferber C., Holovatch Yu. // Phys. Rev E, 1999, vol. 59, p. 6914-6923.

25. Batoulis J., Kremer K. // Macromolecules, 1989, vol. 22, p. 4277.

26. Barret A.J., Tremain D.L. // Macromolecules, 1987, vol. 20, p. 1687.

27. Joanny J.-F., Leibler L., Ball R. // J. Chem. Phys., 1984, vol. 81, p. 4640; Douglas J.F., Freed K.F. // J. Chem. Phys., 1987, vol. 86, p. 4280; Schäfer L., Kappeler Ch. // J. Phys. Paris, 1985, vol. 46, p. 1853; Colloid Polym. Sci., 1990, vol. 268, p. 995.

28. Cates M.E., Witten T.A. // Phys. Rev. Lett., 1986, vol. 56, p. 2497; Phys. Rev. A, 1987, vol. 35, p. 1809-1824.

29. von Ferber C., Holovatch Yu. // Condens. Matter Phys., 1997, No. 10, p. 9-40.

30. von Ferber C., Holovatch Yu. // Europhys. Lett., 1997, vol. 39, p. 31-36.

31. von Ferber C., Holovatch Yu. // Physica A, 1998, vol. 249, p. 327-331.

32. von Ferber C., Holovatch Yu., Schäfer L. // Condens. Matter Phys., 1996, No. 7, p. $15-25$.

33. von Ferber C., Holovatch Yu., Jusufi A., Likos C.N., Löwen H., Watzlawek M. // J. Mol. Phys., 2001, vol. 93, p. 155-158.

34. Likos C.N., Löwen H., Watzlawek M., Abbas B., Jucknischke O., Allgaier J., Richter D. // Phys. Rev. Lett., 1998, vol. 80, p. 4450; Watzlawek M., Likos C.N., Löwen H.

// Phys. Rev. Lett., 1999, vol. 82, p. 5289; Jusufi A., Watzlawek M., Löwen H. // Macromolecules, 1999, vol. 32, p. 4470.

35. Gast A.P. // Langmuir, 1996, vol. 12, p. 4060; Grest G.S., Fetters L.J., Huang J.S., Richter D. // Adv. Chem. Phys., 1996, vol. 94, p. 67.

36. von Ferber C. // Nucl. Phys. B, 1997, vol. 490, p. 511-542.

37. Hentschel H.G.E., Procaccia I. // Physica D, 1983, vol. 8, p. 435-444; Halsey T.C., Jensen M.H., Kadanoff L.P., Procaccia I., Shraiman B.I. // Phys. Rev. A, 1986, vol. 33(2), p. 1141-1151.

38. Burlatsky S.F., Oshanin G.S., Likhachev V.N. // Sov. J. Chem. Phys., 1991, vol. 7, 
p. 1680-1698; Burlatsky S.F., Oshanin G.S. // Phys. Lett. A, 1990, vol. 145, p. 61-65; Oshanin G., Moreau M., Burlatsky S. // Anv. Coll. Int. Sci., 1994, vol. 49, p. 1-46.

39. Witten Jr., T.A., Sander L.M. // Phys. Rev. Lett., 1981, vol. 47, p. 1400-1403; Phys. Rev. A, 1983, vol. 27, p. 5686-5697.

40. Rice S.A. Diffusion-limited reactions. - In: Bamford C.H., Tipper C.F.H., Compton R.G. (eds). Comprehensive Chemical Kinetics. Vol. 25. Amsterdam, Elsevier, 1985.

41. Meakin P. - In: Domb C., Lebowitz J.L. (eds). Phase Transitions and Critical Phenomena. Vol. 12. New York, Academic Press, 1988, p. 336-442; Simulation of Non-equilibrium Growth and Aggregation Processes. Landau D.P., Moon K.K., Schüttler H.-B. (eds). Springer Proceedings in Physics. Computer Studies in Condensed Matter Physics. Vol. 33. Berlin, Springer-Verlag, 1988.

42. von Ferber C., Holovatch Yu. - In: "Renormalization Group'96" 3rd Int. Conf., Dubna, Russia, August 1996, Ed. by: Shirkov D.V., Priezzhev V.B. JINR, Dubna, 1997, p. $123-138$.

43. von Ferber C., Holovatch Yu. // Condens. Matter Phys., 1999, vol. 2, p. 303-312

44. von Ferber C., Holovatch Yu. // J. Mol. Phys., 2001, vol. 93, p. 151-154.

\title{
Теоретико-польовий ренормгруповий аналіз критичних показників зіркових полімерів
}

\author{
К. Фон Фербер ${ }^{1}$, Ю. Головач ${ }^{2,3}$ \\ 1 Теоретична фізика полімерів, Університет Фрайбурґу, \\ D-79104 Фрайбурґ, Німеччина, \\ 2 Інститут фізики конденсованих систем НАН України, \\ 79011 Львів, вул. Свєнціцького, 1 \\ 3 Львівський національний університет ім. І. Франка, \\ 79005 Львів
}

Отримано 18 вересня 2001 р.

Зроблено огляд недавніх результатів аналізу методом теоретикопольової ренормалізаційної групи масштабних (скейлінґових) властивостей зіркових полімерів. Коротко пояснено, як були отримані чисельні значення показників скейлінґу зіркових полімерів. Приведено приклади явищ, які описуються цими показниками. Зокрема, розглядається взаємодія міз зірковими полімерами у доброму розчиннику, бровнівський рух біля полімерного абсорбера, керовані дифузією реакції за участю полімерів.

Ключові слова: зіркові полімери, зіркові показники, ренормалізаційна група

PACS: 64.60.Ak, 61.41.+e, 64.60.Fr, 11.10.Gh 